\title{
The presence of synchronized perfusion dips in the microcirculation of the resting nail bed
}

Robin Mirdell, Aukje Nienke Lemstra-Idsardi, Simon Farnebo and Erik Tesselaar

The self-archived postprint version of this journal article is available at Linköping University Institutional Repository (DiVA):

http:// urn.kb.se/ resolve?urn=urn:nbn:se:liu:diva-153651

N.B.: When citing this work, cite the original publication.

Mirdell, R., Lemstra-Idsardi, A. N., Farnebo, S., Tesselaar, E., (2019), The presence of synchronized perfusion dips in the microcirculation of the resting nail bed, Microvascular Research, 121, 71-81. https:// doi.org/ 10.1016/j.mvr.2018.10.004

Original publication available at:

https:/ / doi.org/ 10.1016/j.mvr.2018.10.004

Copyright: Elsevier

http:// www.elsevier.com/

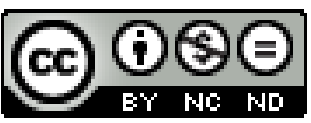




\section{The presence of synchronized perfusion dips in the microcirculation of the resting nail bed}

Robin Mirdell1,3, Aukje Nienke Lemstra-Idsardi' ${ }^{4}$, Simon Farnebo ${ }^{1,3}$, Erik Tesselaar ${ }^{1,2}$.

1. Department of Clinical and Experimental Medicine, Faculty of Health Sciences, Linköping University, Linköping, Sweden

2. Department of Radiation Physics, Department of Medical and Health Sciences, Linköping University, Linköping, Sweden.

3. Department of Plastic Surgery, Hand Surgery, and Burns, Linköping University, Linköping, Sweden

4. University of Twente, Enschede, Netherlands

Corresponding author:

Robin Mirdell

Department of Clinical and Experimental Medicine

Faculty of Health Sciences

Linköping University

SE-58185 Linköping, Sweden

E-mail: robin.mirdell@liu.se 


\section{Abstract}

Objectives: Laser speckle contrast imaging (LSCI) has seen limited use in the study of perfusion dynamics such as vasomotion. The aim of this study was to investigate the effects of a prolonged seated position on perfusion dynamics in the nail bed using LSCI.

Methods: Perfusion was recorded in digits II to IV bilaterally for 20 min during two separate sessions in ten healthy volunteers. The acclimatization period was 5 min for the $1^{\text {st }}$ session and 20 min for the $2^{\text {nd }}$. Perfusion variability and the presence of recurring perfusion dips were analyzed. A digital nerve block was done to verify suspected nervous origin of phenomenon.

Results: Synchronized phases of vasoconstriction were observed in all subjects with perfusion dips in all digits bilaterally and simultaneously. Application of a digital nerve block abolished perfusion dips. The frequency of this phenomenon increased by $25.0 \%$ (95\% CI: 1.6 to $49.2 \%$ ) in the left-hand digits after a prolonged seated position. Perfusion variability increased by $11.6 \%$ (95\% CI: 2.6 to $20.3 \%$ ) in the digits of the left hand. Perfusion changes in right-hand digits did not significantly increase. During the $1^{\text {st }}$ session, temperature increased by $2.7^{\circ} \mathrm{C}(1.1$ to 4.2$)$ while it decreased by $1.3^{\circ} \mathrm{C}(0.2$ to 2.4$)$ during the $2^{\text {nd }}$ session.

Conclusion: The observed perfusion dips are of a centrally mediated nervous origin but are also affected by local factors. They are affected by seating duration and differ between left and right hands, likely because of local micro perfusion dips. This phenomenon seems related to digital thermoregulation.

Keywords: laser speckle contrast imaging, nail bed, microcirculation, perfusion 


\section{List of abbreviations}

LSCI - laser speckle contrast imaging

LDF - laser Doppler flowmetry

PU - perfusion units

AUC - area under the curve 


\section{Introduction}

Microcirculation is generally defined as the smallest order of blood vessels, with a diameter of $<150 \mu \mathrm{m}$, where oxygen and nutrients are exchanged between the blood and the tissue [1, 2]. A functioning microcirculation is vital to any organism and it is therefore an interesting object of study to improve the understanding of both physiology and pathophysiology in many diseases [13]. The microcirculation has an important thermoregulatory function, particularly in the glabrous skin, where a substantial amount of the microcirculatory capacity can be used for shunting of blood to regulate the body's temperature [4].

Many studies on the microcirculation have been done in the skin, likely because of its easy access [1, 5]. Certain skin areas, such as the nailfold, have received particular attention, since the optical conditions and the organization of the microcirculation in the nailfold allow for detailed visualization of blood flow dynamics, for example by using capillary microscopy [6, 7]. Several laser-based techniques have also been used to measure perfusion in the microcirculation to study microvascular physiology and pathology $[1,5,8]$.

In the study of the microcirculation, the concept of vasomotion has received much attention [9-11]. Vasomotion is generally used to describe organized and chaotic fluctuations of vessel diameter primarily occurring in the microvasculature over time $[10,11]$. The presence of this phenomenon was observed for the first time 166 years ago in bats [12]. Traditionally, vasomotion has been investigated using microscopy, different kinds of assays for in-vitro experiments and laser-Doppler flowmetry (LDF) [10, 11]. With increased use of LDF, the concept of flowmetry was introduced. Flowmetry describes the frequency (Doppler) shift of light which has been scattered by moving red blood cells and this correlates to the blood flow in the vessel. The flowmetry signal can then be analyzed to quantify variations in blood flow over time. 
This is slightly different from vasomotion, which strictly speaking is used to describe motion in vessels and not changes in blood flow. [13]. Much effort has been put into investigating different causes for vasomotion and to investigate the physiological implications and functions [10, 11, 1416]. So far, the actual physiological relevance of vasomotion remains uncertain $[10,11]$. Vasomotion is hypothesized to increase tissue perfusion, and this is supported by theoretical work suggesting an increase of blood flow in vessels of oscillatory diameter [14, 15]. However, neither in-vivo nor in-vitro experiments have been able to conclusively verify these suspected benefits with vasomotion in under-perfused tissue $[10,11,16]$.

The mechanisms underlying vasomotion have been studied thoroughly. The current consensus is that vasomotion arises at a local level in arterioles and then propagates through intercellular gap junctions to affect the entire vessel and sometimes adjacent vessels [11, 17-21]. Both calcium channels and the nitric oxide pathway have been implicated as having important mediatory functions and substances affecting these regulatory pathways have been shown to induce vasomotion $[20,22,23]$.

By analyzing the frequency of vasomotion using LDF, different frequency bands have been established which are associated with different types of vasomotion activity [11, 24, 25]. These frequency bands are as follows: cardiac activity $(0.6-1.6 \mathrm{~Hz})$, respiratory activity $(0.15-0.4$ $\mathrm{Hz})$, myogenic activity (0.06-0.15 Hz), neurogenic activity $(0.02-0.06 \mathrm{~Hz})$, and endothelial activity (0.0095-0.02 Hz) [25]. Vascular activity in the different frequency bands can be blocked by affecting the appropriate system [10, 11, 19-23]. Frequencies associated with myogenic activity, neurogenic activity, and endothelial activity are presumed to be of local origin and does not cause synchronized fluctuations in larger parts of the capillary system $[10,11]$. This leaves cardiac activity and respiratory activity responsible for synchronized aspects of vasomotion. 
In addition to the previously described frequency bands, there are two additional phenomena causing synchronized changes in perfusion. The first phenomenon is called Mayer waves, which arise due to a lagging effect in baroreceptors causing an oscillatory effect on perfusion in humans of $0.1 \mathrm{~Hz}[10,11]$. Mayer waves cause synchronized fluctuations and often correspond roughly to the frequency of myogenic activity. The second phenomenon, which has received little attention, consists of synchronized bursts of vasoconstriction through sympathetic innervation occurring simultaneously through the body affecting the shunting of blood through the thermoregulatory parts of the microcirculation, which is primarily localized in glabrous skin of the fingers and toes [4, 26-29].

Previous studies of vasomotion and the microcirculation have used LDF and focused on very small parts of the microcirculation and often on the low frequency spectra related to desynchronized aspects of vasomotion. Laser speckle contrast imaging (LSCI) is a relatively new technique for perfusion measurement $[5,30,31]$ and has seen limited use within the field of vasomotion.

Compared to LDF, which has been the standard method for studying vasomotion, LSCI has the advantage of full field view with high spatial and temporal resolution [1, 5, 30, 31]. This makes it possible to study vasomotion over larger areas and to investigate spatial and temporal synchronicity, which is hard to accomplish using LDF. To our knowledge, LSCI has only been used to study vasomotion in the retina of the mouse [32].

During preliminary experiments in which we measured perfusion in the nailbed, we have observed synchronized bursts of vasoconstriction. In this study, our aim was to further study these burst, referred to as perfusion dips, as well as vasomotion activity in the nail bed, using LSCI. We also investigated the effects of a prolonged seated position and a local nerve block. We 
hypothesized that the number of synchronized vasoconstriction bursts would increase after having maintained a prolonged seated position, and that they would be inhibited by a local nerve block.

\section{Methods}

\section{Study population}

Ten healthy test subjects were recruited, and they gave their informed consent before participation. None of the subjects used regular medication except for three test subjects who used oral contraceptives. None of the participants had any medical conditions to report. One of the test subjects reported sporadic nicotine use. The study was approved by the regional ethics review board, DNr 2012/31/31.

\section{Equipment}

A laser speckle contrast imager (PeriCam PSI System, Perimed AB, Järfälla, Sweden) was used to measure the perfusion in the nail bed. The system has previously been described in detail [33]. LSCI uses a divergent laser and records fluctuations in the speckle pattern, which arises because of the movement of erythrocytes causing blurring during the image integration time [5, 30, 31]. The level of blurriness correlates to the concentration and flow of red blood cells [5, 30, 31]. A typical measurement area ranges from 50 to $400 \mathrm{~cm}^{2}$, where the entire image is captured simultaneously at a frequency of 10-40 Hz. A color-coded image is then created of the measurement area with "red" indicating high perfusion and "blue" low perfusion [5, 30, 31]. Perfusion units (PU) are defined as arbitrary units, similarly to LDF, and system consistency is 
assured by using calibration assays over time [5, 30, 31]. The measurement distance was set to 20-25 cm and the perfusion was recorded using 44 images per second averaged over 4 images with a final frequency of $11 \mathrm{~Hz}$. At the given distance, the resolution was around $0.6 \mathrm{~mm} / \mathrm{speckle}$ pixel.

The temperature of the digits was measured using a one channel thermometer with a temperature probe (TES thermometer 1300, TES electrical electronic corp., Taipei, Taiwan) with a specified accuracy of $0.3 \%$ rdg between $-50{ }^{\circ} \mathrm{C}$ to $199.9^{\circ} \mathrm{C}$ and a resolution of $0.1^{\circ} \mathrm{C}$.

\section{Experimental protocol}

All measurements were conducted in the same room and room temperature was controlled and kept at $22 \pm 1^{\circ} \mathrm{C}$. The experimental procedure was divided into two sessions (see Fig. 1), which were done on different days at least 24 hours apart. In the $1^{\text {st }}$ session, the test subject had remained seated for 5 min prior to the start of the measurement and in the $2^{\text {nd }}$ session, for 20 min. There was no acclimatization period before the test subject was seated. Both forearms were steadily supported, and the hands were placed upon a table in front of them covered by a folded green cloth, with the digits placed at a position slightly below heart level. Care was taken to position the hands at the same position for the repeated measurements. Temperatures of the digits of the left and the right hand were measured by holding the temperature sensor between the pulp of dig I and dig III. Afterwards, both hands were placed close to each other with the dorsal side facing up so that the nail bed of dig II-IV were exposed within a narrow measuring field of $10 \times 8$ cm. Perfusion was measured for 20 min using LSCI. At the end of each session, the temperature of left and right digits was measured once again, in addition to pulse and blood pressure. 


\section{Digital nerve block}

As a separate experiment to verify that the perfusion dips we observed were caused by synchronized bursts of sympathetic nerve activity, a distal digital nerve block was applied to the third digit of the left hand (dig III sin) in one healthy test subject. Mepivacaine (Carbocain, Aspen Nordic, Ballerup, Denmark) at a concentration of $2 \%$ was used and roughly $0.75 \mathrm{ml}$ was injected at each digital nerve. Perfusion was recorded as previously described for 10 min before and 20 min after applying the local anesthesia.

\section{Data analysis}

A dip detection algorithm was designed taking into consideration: the general duration of the dips, the presence of potential motion artifacts, and other contributions to synchronized perfusion changes such as cardiac, respiratory activity and Mayer waves.

The first formula describes the arithmetic mean $(\overline{P U})$ compared to the median $(\widetilde{P U})$ of the raw perfusion value $(P U)$ over a $54.6 \mathrm{~s}$ window corresponding to 600 measurement points. A value higher than 1 indicates a spike in perfusion, likely caused by motion artifacts. Values below 1 are more likely to be caused by the presence of a genuine decrease in perfusion. The formula was designed to describe the predominating type of perfusion activity (a) within a 54.6 second window consisting of perfusion values from both before and after the measurement point $(k)$ in question. 


$$
a=\sum_{k-400}^{k+200} \overline{P U} / \widetilde{P U}
$$

The following formula was then constructed to calculate the approximated perfusion $\left(P U_{a}\right)$ if a perfusion dip event had not occurred. This formula used the $\mathrm{x}^{\text {th }}$ percentile $\left(p_{x}\right)$ calculated with the 'percentil.ink' function in Excel, which uses the linear interpolation between closest ranks method. The exact percentile used was affected by the predominating type of activity $(a)$ accordingly:

$$
\begin{aligned}
& P U(k)>\sum_{k-400}^{k} p_{75 / a^{2}} \rightarrow P U_{a}=P U(k) \\
& P U(k)<\sum_{k-400}^{k} p_{75 / a^{2}} \rightarrow P U_{a}=\sum_{k-200}^{k+200} p_{75 / a^{2}}
\end{aligned}
$$

As a final step, a time window of the actual perfusion was compared to the approximated perfusion $\left(P U_{a}\right)$ without a perfusion dip:

$$
\sum_{k=10}^{k+10} P U / \sum_{k=10}^{k+10} P U_{a}<0.8 \rightarrow \text { perfusion dip }
$$

At this point, the amplitude of the perfusion dip was calculated as the area under the curve $(A U C)$ :

$$
A U C=\sum_{\text {dip start }}^{\text {dip end }} P U_{a}-P U
$$


In addition to the described dip detection, the perfusion variability was also analyzed by using a $36 \mathrm{~s}$ moving time window of the difference between the $75^{\text {th }}$ and the $25^{\text {th }}$ percentile. The mean over the entire 20 min window was then calculated. This variable was intended to reflect the state of vasomotion activity in the microcirculation and is primarily generated by cardiac activity, respiratory activity, and Mayer waves. However, the perfusion variability is also influenced by the presence of perfusion dips and therefore takes all these variables into account describing the total vasomotion activity in an easy way. 


\section{Statistical analysis}

Descriptive statistics of the healthy volunteers are reported as mean followed by the $95 \%$ confidence interval within parentheses. Paired t-tests were done between start and end digital temperature for each session and between left and right-hand digits for all sessions.

Perfusion data between $1^{\text {st }}$ and $2^{\text {nd }}$ session was analyzed using paired t-tests for the following perfusion variable: average AUC, perfusion dip time, average dip amplitude, average perfusion, and variability. A significance level of 0.05 was regarded as significant.

All statistical analyses were made with the aid of Excel 2016 (Microsoft, Redmond Washington USA, www.microsoft.com). 


\section{Results}

An overview of test subject characteristics and temperatures in the digits are given in Table 1. Recurring perfusion dips were observed in all the test subjects with a sudden decrease $(<15 \mathrm{~s})$ of more than $50 \%$ of the measured perfusion value with a quick recovery $(<40$ s) to previous levels.

An overview of the perfusion variables investigated can be seen in Table 2. Figure 2 and 3 show a typical example of a perfusion signal and Figure 4 shows perfusion images from a typical perfusion dip. The average perfusion in both sessions was similar with an average of 164.5 PU (95\% CI: 148.5 to 180.5$)$ in the $1^{\text {st }}$ session and 166.2 PU (95\% CI: 150.9 to 181.6$)$ in the $2^{\text {nd }}$. The average dip amplitude was also similar, 57.6 PU (95\% CI: 51.6 to 63.5) and 56.8 PU (95\% CI: 51.0 to 62.7$)$. The average dip time was $12.9 \%$ (95\% CI: 11.1 to 14.8$)$ during the $1^{\text {st }}$ session and $15.1 \%$ (95\% CI: 13.5 to 16.7$)$ during the $2^{\text {nd }}$ session $(\mathrm{p}=0.055)$. However, in the lefthand digits the effects on the average dip time was more pronounced resulting in a change from $12.4 \%$ (9.8 to 14.9 ) during the $1^{\text {st }}$ session to $15.5 \%$ (13.0 to 17.9$)$ during the $2^{\text {nd }}$ session ( $p=0.047)$. The average variability in both left and right-hand digits changed from 31.3 PU (95\% CI: 29.2 to 33.4 ) during the $1^{\text {st }}$ session to 34.1 PU (95\% CI: 32.2 to 36.1$)$ during the $2^{\text {nd }}$ session $(p=0.007)$. Further analysis of the data also showed the increase of perfusion variability from the first to the second session to be more pronounced in the left hand, while a smaller increase occurred in the right hand which was not significant by itself.

The presence of recurring perfusion dips was completely abolished after application of local anesthesia. The perfusion variables in the nail bed of dig III sin before the digital nerve block were: average AUC 9.7 PU, perfusion dip time 10.9\%, average dip amplitude 89.0 PU, average perfusion 218.8 PU, and perfusion variability 33.2 PU. The perfusion signal can be seen in Figure 5. After the digital nerve block, the same variables were: average AUC 0.0 PU, 
perfusion dip time $0.0 \%$, average dip amplitude 0.0 PU, average perfusion 233.9 PU, and perfusion variability 19.7 PU. All other fingers were unaffected by the digital nerve block and showed similar perfusion characteristics during both measurement windows. The perfusion signal from the second measurement is shown in Figure 6, and Figure 7 shows the perfusion images from a perfusion dip during the second measurement.

Digital temperature between the two different sessions was significantly different. During the $1^{\text {st }}$ session, the digital temperature was $27.4^{\circ} \mathrm{C}$ (95\% CI: 25.5 to 29.3$)$ at the start and increased to $30.1^{\circ} \mathrm{C}$ (95\% CI: 28.2 to 31.9 ) by the end of the session ( $\left.\mathrm{p}=0.003\right)$. In the $2^{\text {nd }}$ session, a temperature decrease was observed from $30.9^{\circ} \mathrm{C}(95 \% \mathrm{CI}: 28.7$ to 33.0$)$ at the start to $29.6^{\circ} \mathrm{C}$ (95\% CI: 27.4 to 31.8) by the end $(\mathrm{p}=0.033)$. The average digital temperature during all sessions was $29.3^{\circ} \mathrm{C}$ (95\% CI: 27.8 to 30.8) in the left hand and $29.3^{\circ} \mathrm{C}$ (95\% CI: 27.9 to 30.8) in the right hand, showing no significant difference between left and right regarding digital temperature $(\mathrm{p}=0.932)$. 


\section{Discussion}

The main finding in this study was the presence of synchronized perfusion dips in the nail bed of all fingers occurring bilaterally and simultaneously. These perfusion dips have not previously been described with imaging techniques and the existence of the phenomenon is seldom mentioned. No previous attempts have been done to quantify perfusion dip amplitude and frequency in a population of healthy test subjects. Application of a digital nerve block completely abolished the perfusion dips in the affected finger. These results suggest a neurogenic origin of the phenomenon. These findings support earlier descriptions of sympathetic innervation of arteriovenous shunts primarily present in the microcirculation of glabrous skin [26-29]. The synchronicity of the phenomenon and its temporal aspects have now been described clearly, which was not possible without imaging techniques such as LSCI. This study shows several of the benefits with LSCI when studying microcirculatory phenomena when both spatial and temporal resolution is of high importance.

A simple provocation in form of a prolonged seated position resulted in a slight increase of perfusion dips in the left hand of the test subjects but did not cause a significant change in the perfusion dips of the right hand. The perfusion variability was also measured, which uses the average difference between the $75^{\text {th }}$ and the $25^{\text {th }}$ percentile over a moving time window. This variable is primarily affected by cardiogenic and respiratory activity, but also by the frequency and amplitude of perfusion dips. Generally, perfusion variability should be viewed as a marker for vasomotion activity capturing many aspects of both synchronized and asynchronous vasomotion occurring at a systemic level. Perfusion variability was significantly increased in the nail bed after a prolonged seated position, but this increase was only significant for the left hand when sub analysis was conducted. 
In the $1^{\text {st }}$ session, the digital temperature increased by $2.7^{\circ} \mathrm{C}$, while it decreased by $1.3^{\circ} \mathrm{C}$ during the $2^{\text {nd }}$ session. At the end of the $1^{\text {st }}$ session the temperature was $30.1^{\circ} \mathrm{C}$ (95\% CI: 28.231.9), which was quite similar to the temperature at the start of the $2^{\text {nd }}$ session, $30.9^{\circ} \mathrm{C}(95 \% \mathrm{CI}$ : 28.7-33.0). These two measurement points corresponds to $25 \mathrm{~min}$ and 20 min after assuming a seated position, respectively. This suggests that digital temperature first increases when assuming a seated position and then starts to decline at some point within the first 25 min. These changes in digital temperature are likely linked to the observed variations in perfusion dynamics. The initial increase in temperature would occur in conjunction with a vasodilatory response, decreasing the likeliness of perfusion dips occurring. As a prolonged seated position is maintained, digital temperature will start to decrease due to a simultaneous increase in vasoconstrictive activity, therefore increasing the likelihood of perfusion dips. As the frequency of the perfusion dips increases, the total effect on the digital perfusion becomes greater and the temperature of the digits decrease as a result.

Similar changes in skin perfusion have previously been described in the lower extremity during this time frame [34], showing a decrease in skin temperature over 30 min after 20 min of acclimatization. Although we measured the perfusion in the nailbed, it seems intricately connected to the perfusion in the skin, which can be seen in Figures 4 and 7 where a clear perfusion decrease is observed in the entire hand during the perfusion dips. It is likely, however, that the perfusion dips in the skin are contained primarily to the glabrous skin of the digits because of the rich presence of arteriovenous shunts.

Microcirculatory differences were also noted between the digits of left and right hand in the present study. Previous work has shown that there are microcirculatory differences in other parts of the body as well, such as the microcirculation of the face, when vasomotion has been 
studied using LDF [35]. This raises a few questions, particularly since the presence of perfusion dips seem to be centrally mediated while there also is a local difference between anatomical sites. The most reasonable explanation for this would be that the perfusion dips are affected by local processes in addition to the centrally mediated drive. Some perfusion dips would therefore be elicited slightly earlier and for a longer duration with little impact on the maximal amplitude. These viewpoints would also explain the presence of micro perfusion dips with a smaller amplitude, which we observed in individual digits with a less pronounced perfusion change in other digits.

In many studies investigating vasomotion, a wavelet transform is often performed due to its value in detecting perfusion changes at specific frequency, particularly of the lower frequencies. However, due to the varying period of the perfusion dips and their individual shape, as can be seen in Figures 2, 3, 5, and 6, the spectral density would be smeared-out over a too large frequency span to generate any interesting data. This is in contrast to the otherwise welldefined frequency spans of vasomotion described with LDF. As many of the low frequency aspects of vasomotion are considered asynchronous [10,11], they will not be measurable with LSCI because the perfusion data is averaged over a relatively large area. Taking these factors into account, we decided that a wavelet transform would contribute little to the results of the study.

This study otherwise has several limitations. No sample size analysis was done in the planning stage of the study because of its exploratory nature. For this reason, only 10 healthy test subjects were included; making the study underpowered to detect significant changes among many of the tested variables. The digital nerve block was also only applied in one test subject, which is a substantial limitation even though the data presented in Figures 5-7 appears convincing in proving the nervous origin of the perfusion dips in the individual in question. 
Another limitation was the lack of a control substance in the contralateral digit. More studies of vasomotion using LSCI are therefore needed to alleviate some of these concerns.

The formula used for perfusion dip detection in the present work succeeded at its task, but never went through a formal optimization process. This is obviously an area for further research. Before such work is undertaken, perfusion dip patterns should preferably be investigated in pathological conditions affecting the microcirculation of the digits. Such data would facilitate further development of the optimal mathematical approach to perfusion dip detection and how to discern between normal and pathological conditions.

During the study, it was noted that perfusion dips could be induced voluntarily. This was tested in two separate subjects in measurements not included in the study data. Both subjects were able to induce perfusion dips on instruction through either thinking about moving their hands or by use of respiratory exercises, such as taking a deep breath followed by slowly exhaling. However, both subjects were unable to induce a new perfusion dip until at least a few minutes had passed since the last induction. This information was kept from all test subjects in the study because it could otherwise have created a source of error. Nonetheless, this finding shows interesting insights into centrally mediated microcirculatory mechanisms of the digits, possibly connected to their thermoregulatory functions.

Interesting areas of further research would be to investigate the presence or the lack of perfusion dips in patients with known thermoregulatory disturbances of the digits, such as patients with Raynaud's phenomenon or systemic sclerosis [26]. An overactive vasoconstrictive activity with a lack of dynamic alterations between vasodilation and vasoconstriction might be an important part of the pathogenesis. LSCI seems particularly suited to further investigate these 
aspects with its possibility to record perfusion in both hands simultaneously while also providing excellent temporal resolution.

Another interesting field of investigation would be metabolic diseases such as diabetes to study the development of neuropathies. The absence of perfusion dips could potentially be an early sign of conditions to come and might be used as an objective method to evaluate generalized neuropathies. To use LSCI for perfusion measurement could also be an interesting complementary method to investigating nerve damage of traumatic or iatrogenic origin. Finally, it could potentially be used to objectively evaluate the effects of nerve blocks with local anesthesia.

LSCI as a method for objective perfusion measurement has the advantage of both excellent spatial and temporal resolution [5, 30, 31]. This makes the method particularly suited for studying perfusion dynamics over large areas. It also has the advantage of being completely non-invasive without any skin contact, making it a useful method for the study of synchronized aspects of vasomotion in many diseases of vascular origin where many microcirculatory questions remain. 


\section{Conclusion}

Perfusion recordings with LSCI showed the presence of synchronized bursts of vasoconstriction causing perfusion dips in the nail bed of healthy test subjects. After a prolonged seated position these perfusion dips occurred more frequently in the left-hand digits but not in the right-hand digits. Changes in perfusion variability were also observed, with a more pronounced effect in the left-hand digits. A digital nerve block abolished the presence of the phenomenon. This suggests a centrally mediated origin which also seem affected by local factors. After a prolonged seated position, a slight temperature decrease was observed, and it was associated with an increase in perfusion dynamics. The described perfusion phenomenon is likely connected to thermoregulatory properties of the digits. 


\section{Conflict of interest}

The authors declare that they have no conflict of interest. 


\section{References}

[1] Eriksson S., Nilsson J., Sturesson C. Non-invasive imaging of microcirculation: a technology review. Med Devices (Auckl). 2014 Dec 9;7:445-52. doi: 10.2147/MDER.S51426.

[2] Jung F., Leithäuser B., Landgraf H., Jünger M., Franzeck U., Pries A. et al. Laser Doppler flux measurement for the assessment of cutaneous microcirculatio--critical remarks. Clin Hemorheol Microcirc. 2013 Jan 1;55(4):411-6. doi: 10.3233/CH-131778.

[3] Boerma E.C. The microcirculation as a clinical concept: work in progress. Curr Opin Crit Care. 2009 Jun;15(3):261-5. doi: 10.1097/MCC.0b013e32832b0ff4.

[4] Romanovsky AA. Skin temperature: its role in thermoregulation. Acta Physiol (Oxf). 2014 Mar;210(3):498-507.

[5] Briers J.D. Laser Doppler, speckle and related techniques for blood perfusion mapping and imaging. Physiol. Meas. 2001 Nov;22(4):R35-66.

[6] Ingegnoli F., Gualtierotti R. A systematic overview on the use and relevance of capillaroscopy in systemic sclerosis. Expert Rev Clin Immunol. 2013 Nov;9(11):1091-7. doi: 10.1586/1744666X.2013.849198.

[7] Ingegnoli F, Smith V, Sulli A, Cutolo M. Capillaroscopy in Routine Diagnostics: Potentials and Limitations. Curr Rheumatol Rev. 2018 Apr 20;14(1):5-11.

[8] Hu HF, Hsiu H, Sung CJ, Lee CH. Combining laser-Doppler flowmetry measurements with spectral analysis to study different microcirculatory effects in human prediabetic and diabetic subjects. Lasers Med Sci. 2017 Feb;32(2):327-334.

[9] Chambers R. Vasomotion in the hemodynamics of the blood capillary circulation. Ann N Y Acad Sci. 1948;49(Art 4):549-52.

[10] Nilsson H, Aalkjaer C. Vasomotion: mechanisms and physiological importance. Mol Interv. 2003 Mar;3(2):79-89, 51.

[11] Aalkjær C., Boedtkjer D., Matchkov V. Vasomotion - what is currently thought? Acta Physiol (Oxf). 2011 Jul;202(3):253-69. doi: 10.1111/j.1748-1716.2011.02320.x.

[12] Jones, T.W. Discovery that the veins of the bat's wing are endowed with rhythmical contractility and that onward flow of blood is accelerated by each contraction. Phil. Trans. Roy. Soc. Lond. 1852 Jan;142:131-136.

[13] Intaglietta M. Vasomotion and flowmotion: physiological mechanisms and clinical evidence. Vascular Medicine Review 1990;1:101-112.

[14] Ursino M., Cavalcanti S., Bertuglia S., Colantuoni A. Theoretical analysis of complex oscillations in multibranched microvascular networks. Microvasc Res. 1996 Mar;51(2):229-49. 
[15] Tsai A.G., Intaglietta M. Evidence of flowmotion induced changes in local tissue oxygenation. Int J Microcirc Clin Exp. 1993 Feb;12(1):75-88.

[16] Rücker M., Strobel O., Vollmar B., Roesken F., Menger M.D. Vasomotion in critically perfused muscle protects adjacent tissues from capillary perfusion failure. Am J Physiol Heart Circ Physiol. 2000 Aug;279(2):H550-8.

[17] Porret C.A., Stergiopulos N., Hayoz D., Brunner H.R., Meister J.J. Simultaneous ipsilateral and contralateral measurements of vasomotion in conduit arteries of human upper limbs. Am J Physiol. 1995 Dec;269(6 Pt 2):H1852-8.

[18] Wilkin JK. Periodic cutaneous blood flow during postocclusive reactive hyperemia. Am J Physiol. 1986 May;250(5 Pt 2):H765-8.

[19] Gokina N.I., Bevan R.D., Walters C.L., Bevan J.A. Electrical activity underlying rhythmic contraction in human pial arteries. Circ Res. 1996 Jan;78(1):148-53.

[20] Hill C.E., Eade J., Sandow S.L. Mechanisms underlying spontaneous rhythmical contractions in irideal arterioles of the rat. J Physiol. 1999 Dec 1;521 Pt 2:507-16.

[21] Peng H., Matchkov V., Ivarsen A., Aalkjaer C., Nilsson H. Hypothesis for the initiation of vasomotion. Circ Res. 2001 Apr 27;88(8):810-5.

[22] Bertuglia S., Colantuoni A., Intaglietta M. Effects of L-NMMA and indomethacin on arteriolar vasomotion in skeletal muscle microcirculation of conscious and anesthetized hamsters. Microvasc Res. 1994 Jul;48(1):68-84.

[23] Kasai Y., Yamazawa T., Sakurai T., Taketani Y., Iino M. Endothelium-dependent frequency modulation of $\mathrm{Ca} 2+$ signalling in individual vascular smooth muscle cells of the rat. J Physiol. 1997 Oct 15;504 (Pt 2):349-57.

[24] Söderström T., Stefanovska A., Veber M., Svensson H. Involvement of sympathetic nerve activity in skin blood flow oscillations in humans. Am J Physiol Heart Circ Physiol. 2003 May;284(5):H1638-46.

[25] Lal C., Unni S.N. Correlation analysis of laser Doppler flowmetry signals: a potential noninvasive tool to assess microcirculatory changes in diabetes mellitus. Med Biol Eng Comput. 2015 Jun;53(6):557-66. doi: 10.1007/s11517-015-1266-y.

[26] Flavahan NA. A vascular mechanistic approach to understanding Raynaud phenomenon. Nat Rev Rheumatol. 2015 Mar;11(3):146-58. doi: 10.1038/nrrheum.2014.195.

[27] Bergersen, T. K., Eriksen, M., Walloe, L. Local constriction of arteriovenous anastomoses in the cooled finger. Am. J. Physiol. 273, R880-R886 (1997).

[28] Bini, G., Hagbarth, K. E., Hynninen, P. \& Wallin, B. G. Regional similarities and differences in thermoregulatory vaso- and sudomotor tone. J. Physiol. 306, 553-565 (1980). 
[29] Bini, G., Hagbarth, K. E., Hynninen, P. \& Wallin, B. G. Thermoregulatory and rhythmgenerating mechanisms governing the sudomotor and vasoconstrictor outflow in human cutaneous nerves. J. Physiol. 306, 537-552 (1980).

[30] Boas D. A, Dunn A.K. Laser speckle contrast imaging in biomedical optics. J Biomed Opt. 2010 Jan-Feb;15(1):011109. doi: 10.1117/1.3285504

[31] Basak K, Manjunatha M, Dutta PK. Review of laser speckle-based analysis in medical imaging. Med Biol Eng Comput. 2012 Jun;50(6):547-58. doi:10.1007/s11517-012-0902-z.

[32] Neganova AY., Postnov DD., Sosnovtseva O., Jacobsen JC. Rat retinal vasomotion assessed by laser speckle imaging. PLoS One. 2017 Mar 24;12(3):e0173805. doi:

10.1371/journal.pone.0173805.

[33] Lindahl F, Tesselaar E, Sjöberg F. Assessing paediatric scald injuries using laser speckle contrast imaging. Burns. 2013;39:662-6. doi: 10.1016/j.burns.2012.09.018.

[34] Namkoong S, Shim J, Kim S, Shim J. Effects of different sitting positions on skin temperature of the lower extremity. J Phys Ther Sci. 2015 Aug;27(8):2637-40. doi: 10.1589/jpts.27.2637.

[35] Benedicic M., Bernjak A., Stefanovska A., Bosnjak R. Continuous wavelet transform of laser-Doppler signals from facial microcirculation reveals vasomotion asymmetry. Microvasc Res. 2007 Jul;74(1):45-50. 


\section{Figure texts}

Figure 1. Overview of the experimental protocol. The two measurement sessions were held with an interval of at least 24 hours. LSCI: laser speckle contrast imaging; temp: measurement of temperature in the pulp of digits I and III.

\begin{tabular}{|c|c|c|c|c|c|}
\hline Time (min) & 0 & $5 \mathrm{~min}$ & $20 \mathrm{~min}$ & $25 \mathrm{~min}$ & $40 \mathrm{~min}$ \\
\hline \multirow[t]{2}{*}{ 1st session } & Acclimatization & LSCImeasurement & & \multirow[b]{2}{*}{ temp } & \\
\hline & & temp & & & \\
\hline \multirow[t]{2}{*}{ 2nd session } & Acclimatization & & LSCI me & & \multirow[b]{2}{*}{ temp } \\
\hline & & & temp & & \\
\hline
\end{tabular}


Figure 2. Typical perfusion signal from the nail bed during the $1^{\text {st }}$ session. The dark blue line in the top graph shows the $4 \mathrm{~s}$ moving average of the perfusion signal from dig II sin. The dark blue line in the middle graph shows the perfusion signal from dig II $\mathrm{dx}$. The orange lines and the grey lines in the top and middle graph show detected perfusion dips and area under the curve (AUC), respectively. The bottom graph shows the $4 \mathrm{~s}$ moving average of the perfusion signal from each digit with: dark blue = dig II dx, orange = dig II sin, green = dig III dx, yellow = dig III sin, light blue $=\operatorname{dig} \mathrm{IV} \mathrm{dx}$, and green $=\operatorname{dig} \mathrm{IV} \sin$.

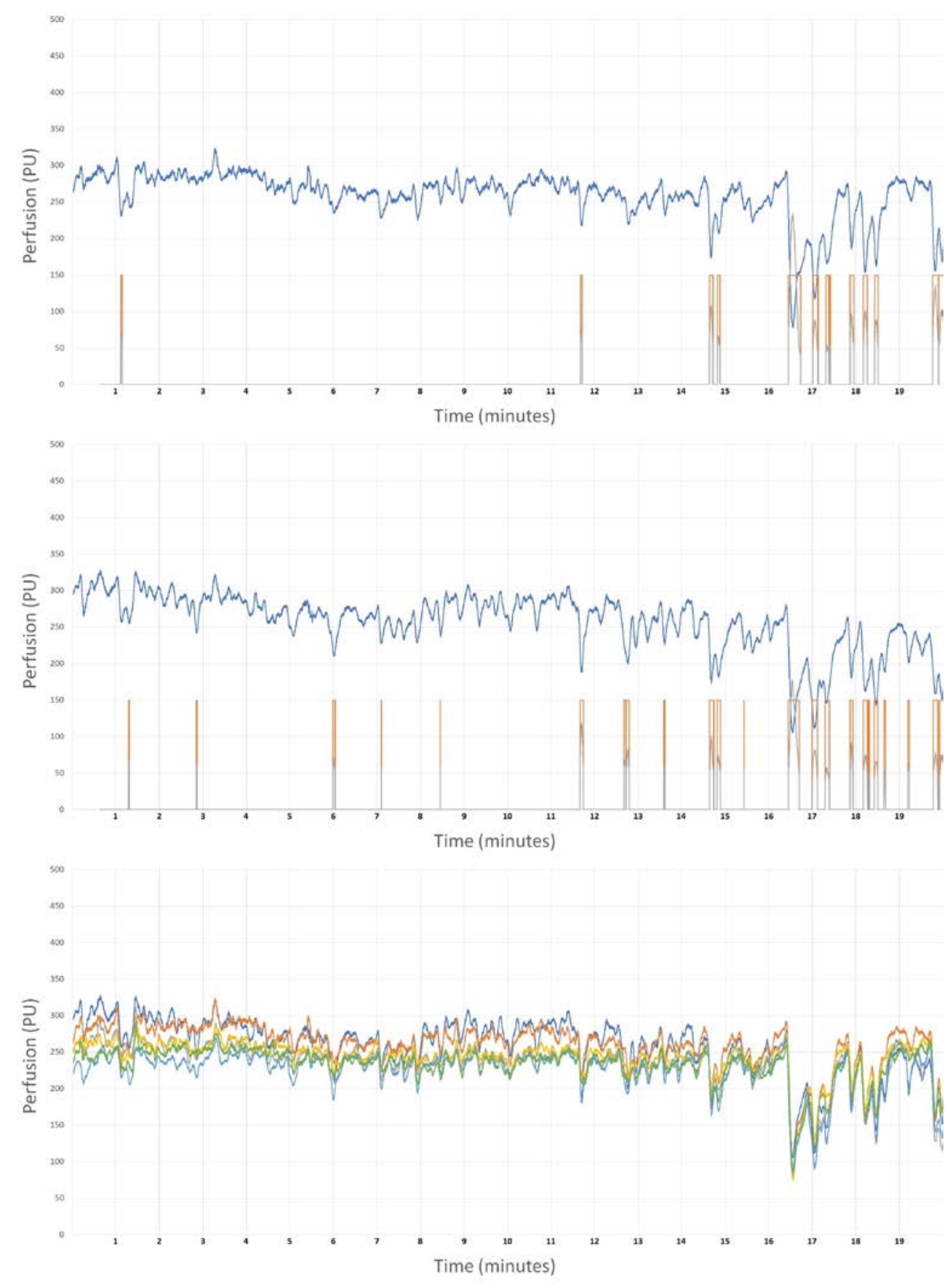


Figure 3. Typical perfusion signal from the nail bed during the $2^{\text {nd }}$ session. The dark blue line in the top graph shows the $4 \mathrm{~s}$ moving average of the perfusion signal from dig II sin. The dark blue line in the middle graph shows the perfusion signal from dig II dx. The orange lines and the grey lines in the top and middle graph show detected perfusion dips and area under the curve (AUC), respectively. The bottom graph shows the $4 \mathrm{~s}$ moving average of the perfusion signal from each digit with: dark blue = dig II dx, orange = dig II sin, green = dig III dx, yellow = dig III sin, light blue = dig IV dx, and green = dig IV sin .

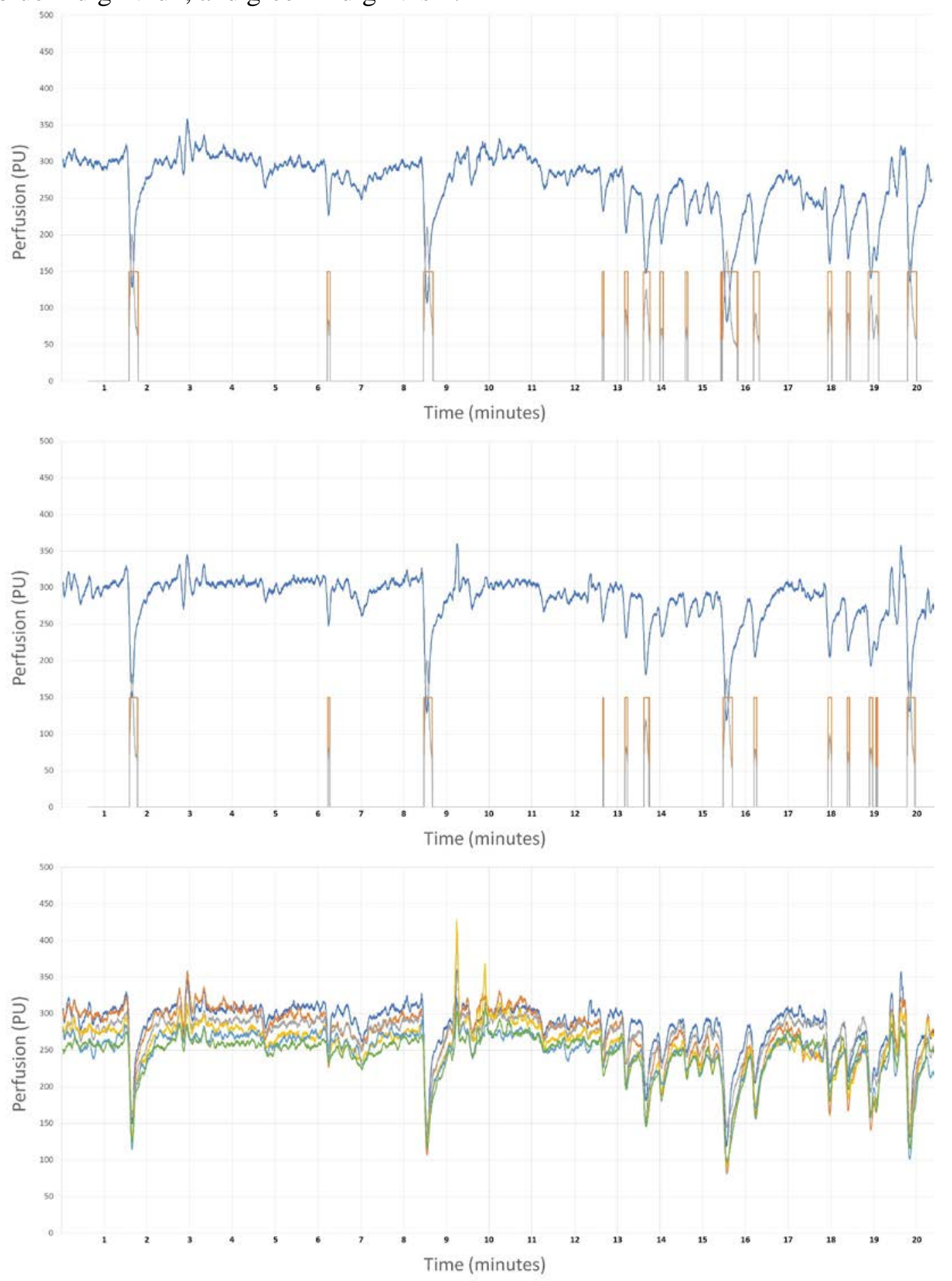


Figure 4. Perfusion images captured $15 \mathrm{~min}$ after the start of the $2^{\text {nd }}$ session in the most representative test subject, from the same recording as the perfusion signal in Figure 3. The left perfusion image shows both hands in a vasodilatory state $15 \mathrm{~min}$ and $20 \mathrm{~s}$ after the start of the recording. The perfusion image to the right is captured $13 \mathrm{~s}$ later and shows a clear decrease in perfusion in all digits. The white numbered circles are the regions of interest for each nailbed perfusion signal.

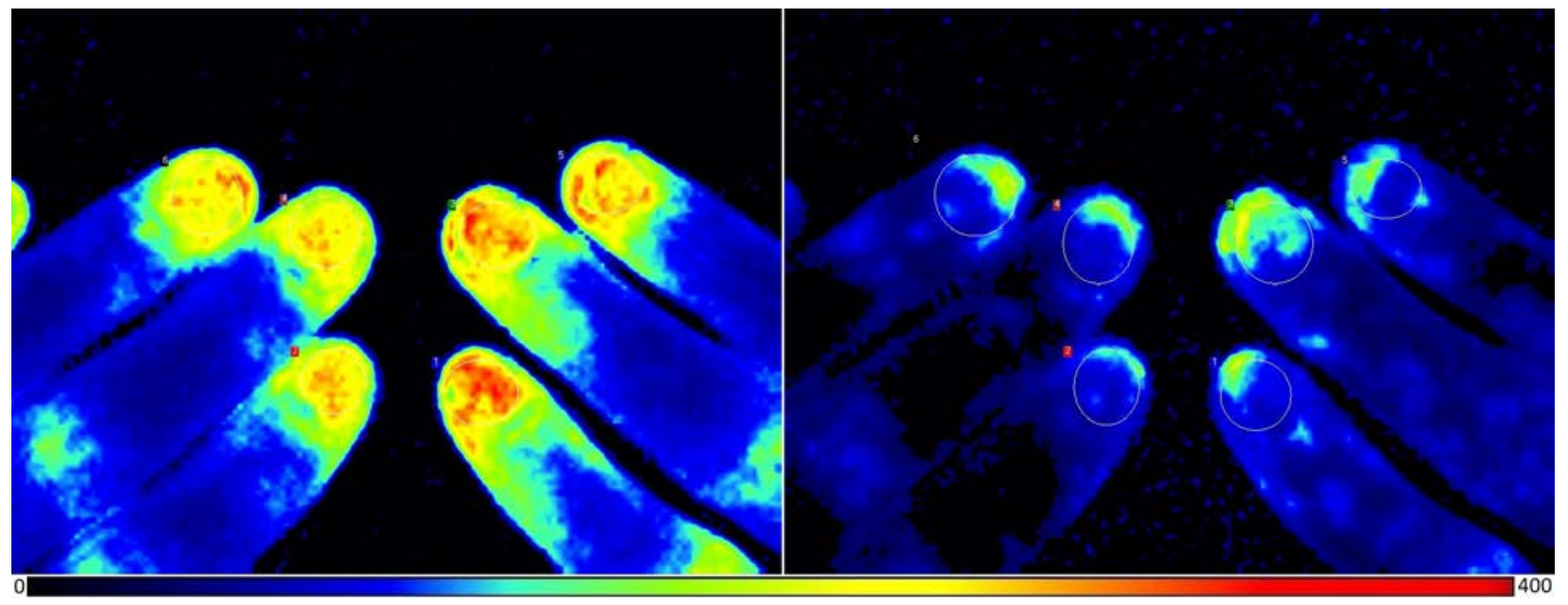


Figure 5. Typical perfusion signal from the nail bed with detected perfusion dips before application of digital nerve block. The dark blue line in the top graph shows the $4 \mathrm{~s}$ moving average of the perfusion in dig III sin. The dark blue line in the middle graph shows the raw perfusion signal recorded at $11 \mathrm{~Hz}$. The orange lines and the grey lines in the top and middle graph show detected perfusion dips and area under the curve (AUC), respectively. The bottom graph shows the $4 \mathrm{~s}$ moving average of the perfusion in each digit with: dark blue $=\mathrm{dig} \mathrm{II} \mathrm{dx}$, orange = dig II sin, green = dig III dx, yellow = dig III sin, light blue = dig IV dx, and green = dig IV sin.

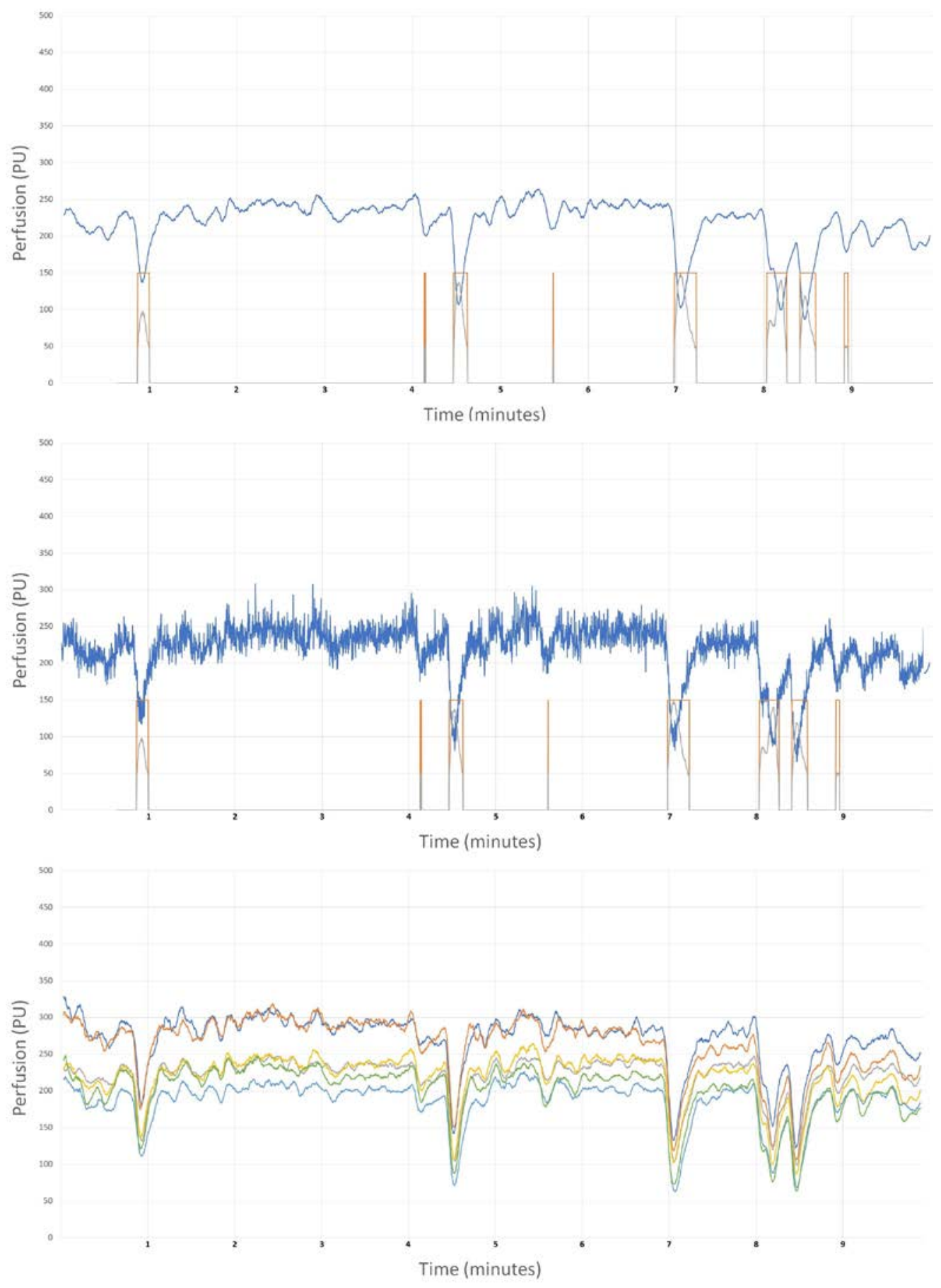


Figure 6. Typical perfusion signal from the nail bed with detected perfusion dips 5 min after application of digital nerve block to dig III sin. The dark blue line in the top graph shows the $4 \mathrm{~s}$ moving average of the perfusion in dig III sin. The dark blue line in the middle graph shows the perfusion in dig III dx, unaffected by the digital nerve block. The orange lines and the grey lines in the middle graph show detected perfusion dips and area under the curve (AUC), respectively. The bottom graph shows the $4 \mathrm{~s}$ moving average of the perfusion in each digit with: dark blue = dig II dx, orange = dig II sin, green = dig III dx, yellow = dig III sin, light blue = dig IV dx, and green $=\operatorname{dig} I V \sin$.

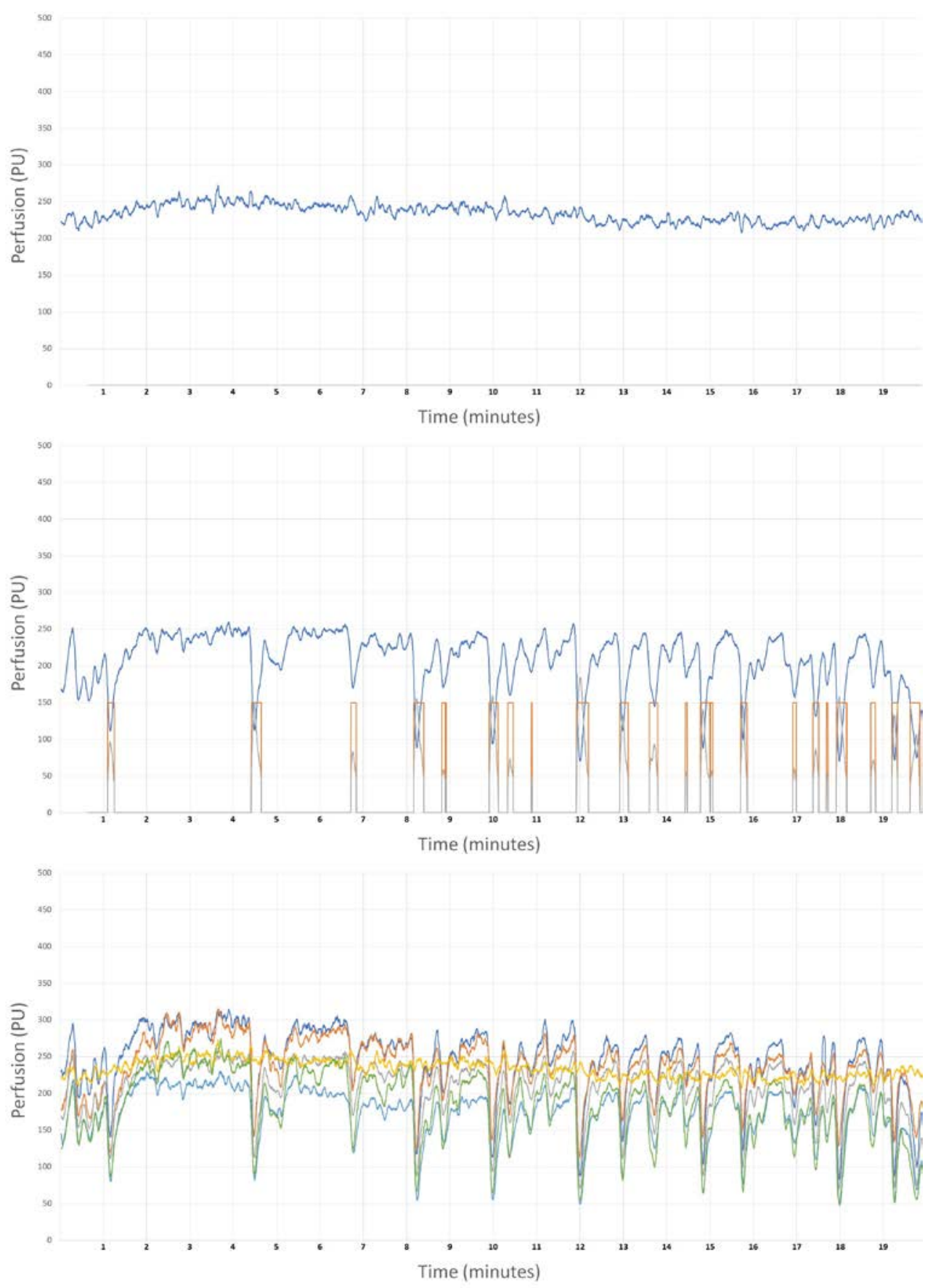


Figure 7. Perfusion images captured 17 min after the application of digital nerve block on dig III sin, from the same recording as the perfusion signal in Figure 6. The left perfusion image shows both hands in a vasodilatory state $11 \mathrm{~min}$ and $56 \mathrm{~s}$ after the start of the recording. The perfusion image to the right is captured $9 \mathrm{~s}$ later and shows a marked decrease in perfusion in all digits except for dig III sin, which is affected by the digital nerve block. The white numbered circles are the regions of interest for each nailbed perfusion signal.

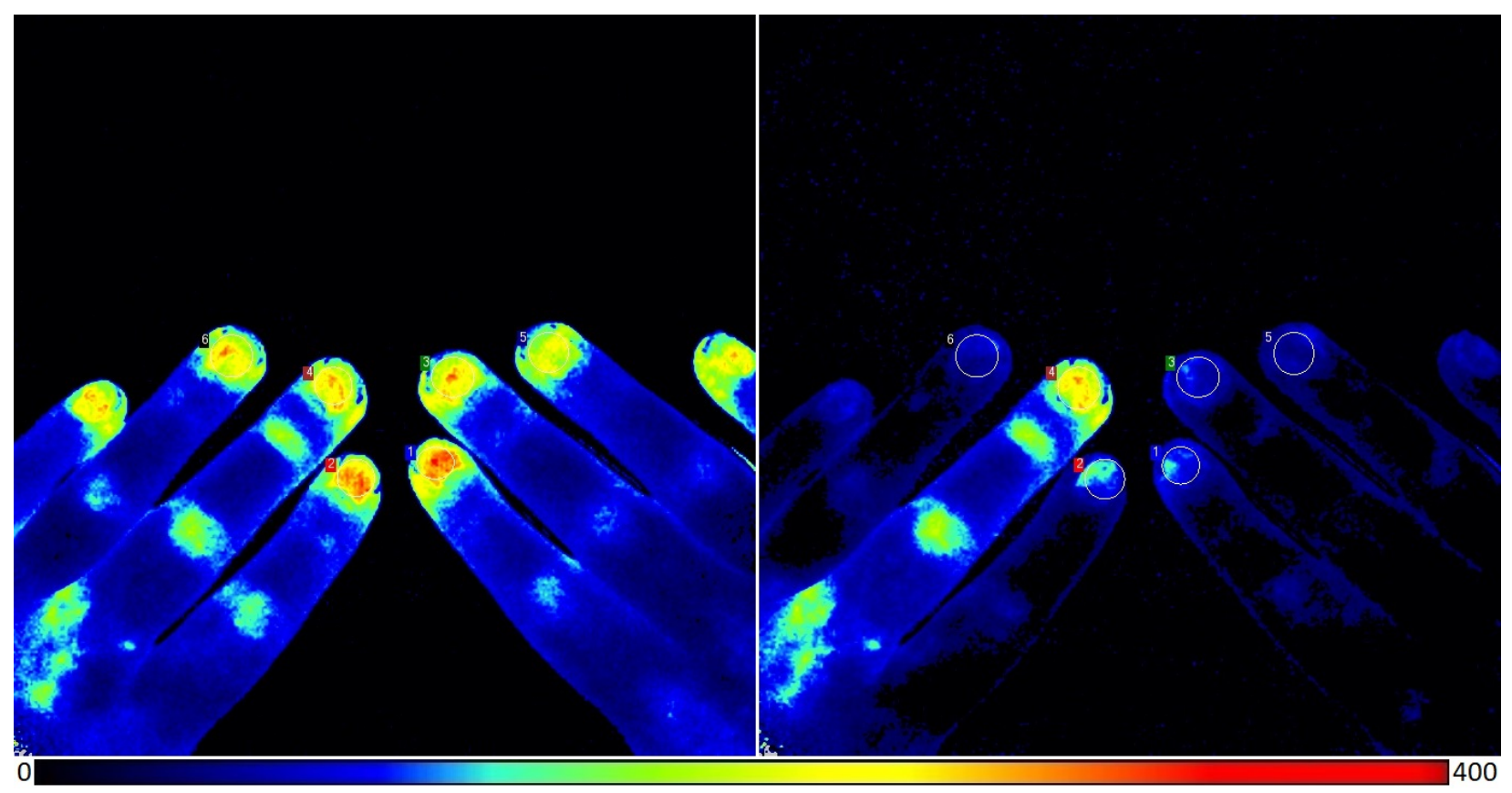




\section{Table texts}

Table 1. Descriptive statistics of the population in the study. All values are reported as mean (95\% CI) unless otherwise specified. Significant differences between sessions are marked using stars. ${ }^{*} \mathrm{p}=0.033{ }^{* *} \mathrm{p}=0.003$

\begin{tabular}{|c|c|}
\hline Sex & 7 females and 3 males \\
\hline Age & 26.0 years (23.5 to 28.5 ) \\
\hline Weight & $68.5 \mathrm{~kg}(62.3$ to 74.7$)$ \\
\hline Length & $170.7 \mathrm{~cm} \mathrm{(165.4} \mathrm{to} 176.0)$ \\
\hline Saturation $\left(\mathrm{SpO}_{2}\right)$ & $98.3 \%$ (97.5 to 99.1$)$ \\
\hline Nicotine use & 1 out of 10 \\
\hline Medication usage & 3 out of 10 \\
\hline $\begin{array}{l}\text { Caffeine last } 24 \text { hours, } 1^{\text {st }} \\
\text { session and } 2^{\text {nd }} \text { session }\end{array}$ & 7 out of 10 , and 4 out of 10 \\
\hline $\begin{array}{l}\text { Physical activity last } 24 \text { hours, } \\
1^{\text {st }} \text { session and } 2^{\text {nd }} \text { session }\end{array}$ & 1 out of 10 , and 3 out of 10 \\
\hline $\begin{array}{l}\text { Systolic blood pressure, } 1^{\text {st }} \\
\text { session and } 2^{\text {nd }} \text { session }\end{array}$ & $113 \mathrm{mmHg}$ (109 to 118 ), and $112 \mathrm{mmHg}$ (107 to 117 ) \\
\hline $\begin{array}{l}\text { Diastolic blood pressure, } 1^{\text {st }} \\
\text { session and } 2^{\text {nd }} \text { session }\end{array}$ & $72 \mathrm{mmHg}$ (67 to 77 ), and $72 \mathrm{mmHg}$ (67 to 78 ) \\
\hline Pulse, $1^{\text {st }}$ session and $2^{\text {nd }}$ session & $65.0 \mathrm{bpm}$ (59.3 to 70.3), and $67.0 \mathrm{bpm}$ (60.7 to 73.3) \\
\hline $\begin{array}{l}\text { Digital temperature, at start and } \\
\text { end of } 1^{\text {st }} \text { session }\end{array}$ & $27.4^{\circ} \mathrm{C}$ (25.5 to 29.3$)$, and $30.1^{\circ} \mathrm{C}(28.2$ to 31.9$) * *$ \\
\hline $\begin{array}{l}\text { Digital temperature, at start and } \\
\text { end of } 2^{\text {nd }} \text { session }\end{array}$ & $30.9^{\circ} \mathrm{C}(28.7$ to 33.0$)$, and $29.6^{\circ} \mathrm{C}(27.4$ to 31.8$) *$ \\
\hline $\begin{array}{l}\text { Digital temperature of left and } \\
\text { right digits for all sessions }\end{array}$ & $29.3^{\circ} \mathrm{C}(27.8$ to 30.8$)$, and $29.3^{\circ} \mathrm{C}$ (27.9 to 30.8$)$ \\
\hline
\end{tabular}


Table 2. Perfusion variables measured during the $1^{\text {st }}$ and $2^{\text {nd }}$ sessions and average differences in perfusion variables between $2^{\text {nd }}$ and $1^{\text {st }}$ session as mean difference ( $\left.95 \% \mathrm{CI}\right)$. A positive number indicates an increase in the $2^{\text {nd }}$ session compared to the $1^{\text {st }}$ session. ${ }^{*} \mathrm{p}=0.047{ }^{*+} \mathrm{p}=0.018$ ** $\mathrm{p}=0.007$

\begin{tabular}{|c|c|c|c|}
\hline Session 1 & Left & Right & Total \\
\hline Average AUC (PU) & $6.6(5.0$ to 8.3$)$ & $7.4(5.7$ to 9.1$)$ & 7.0 (5.8 to 8.2$)$ \\
\hline Time (\%) & $12.4(9.8$ to 14.9$)$ & 13.5 (10.7 to 16.4$)$ & 12.9 (11.1 to 14.8$)$ \\
\hline Average dip amplitude (PU) & 57.2 (49.1 to 65.2$)$ & 57.9 (49.1 to 66.8$)$ & 57.6 (51.6 to 63.5$)$ \\
\hline Average perfusion (PU) & 166.9 (144.5 to 189.3$)$ & 162.1 (138.9 to 185.3$)$ & 164.5 (148.5 to 180.5$)$ \\
\hline Variability $(P U)$ & 31.1 (28.3 to 34.0$)$ & 31.5 (28.4 to 34.6$)$ & 31.3 (29.2 to 33.4$)$ \\
\hline
\end{tabular}

Session 2
Average AUC (PU)
Time (\%)
Average dip amplitude (PU)
Average perfusion $(P U)$
Variability (PU)

Left

Right

Total

\begin{tabular}{|lll|}
\hline $8.3(6.7$ to 9.9$)$ & $7.7(6.5$ to 8.9$)$ & $8.0(7.0$ to 9.0$)$ \\
\hline $15.5(13.0$ to 17.9$)$ & $14.6(12.5$ to 16.8$)$ & $15.1(13.5$ to 16.7$)$ \\
\hline $56.0(48.2$ to 63.9$)$ & $57.7(49.0$ to 66.4$)$ & $56.8(51.0$ to 62.7$)$ \\
\hline $164.8(143.7$ to 185.9$)$ & $167.7(145.1$ to 190.3$)$ & $166.2(150.9$ to 181.6$)$ \\
$34.7(31.7$ to 37.6$)$ & $33.6(31.0$ to 36.2$)$ & $34.1(32.2$ to 36.1$)$
\end{tabular}

Session differences

Left

Right

Total

Average AUC (PU)
Time (\%)
Average dip amplitude (PU)
Average perfusion (PU)
Variability (PU)

1.7

\begin{tabular}{lll}
\hline $.7(0.0$ to 3.4$)$ & $0.3(-1.3$ to 1.8$)$ & $1.0(-0.2$ to 2.2$)$ \\
$3.1(0.2$ to 6.1$) *$ & $1.1(-1.9$ to 4.1$)$ & $2.1(0.0$ to 4.2$)$ \\
$-1.1(-5.2$ to 2.9$)$ & $-0.3(-5.4$ to 4.9$)$ & $-0.7(-3.9$ to 2.5$)$ \\
$-2.1(-16.9$ to 12.7$)$ & $5.6(-8.8$ to 20.0$)$ & $1.7(-8.5$ to 12.0$)$ \\
$3.5(0.8$ to 6.3$){ }^{*+}$ & $2.1(-0.7$ to 4.9$)$ & $2.8(0.9$ to 4.8$) * *$
\end{tabular}

